\title{
New Possibilities of Using Microscopic Techniques in Forensic Field
}

\author{
Marek Kotrlý*1,2 \\ 1. Institute of Criminalistics Prague (ICP), Czech Republic; \\ 2. Charles University in Prague, Faculty of Science, Praha, Czech Republic.
}

Introduction

Techniques of electron microscopy are widely applied in a forensic field, both for an initial screening and a final expert assessment. The set of materials that are delivered to a forensic lab is very extensive, practically any material produced by human and nature activities relating to the case that is examined can be encountered (ranging from the fragment of an ancient vessel, data records, documents, to hightech semiconductors). Therefore, materials of organic origin, plants and animal fragments, etc., are investigated as well.

\section{Methodology}

Electron microscopy combined with EDS/WDS is currently employed e.g. for analyses of these materials: a) unknown samples (including powders from extortionate letters, etc.); b) mineralogical, petrological and gemmological objects (mineral relics, soils, precious stones and their imitations, etc.); c) gunshot residues (GSR); d) explosives, propellants and fulminating compounds; e) post-blast residues (PBR), and other thermogenetic particles; f) fillers and additives of paper and plastics; g) pigments and paint systems, including colour layers of artworks; h) cosmetic and pharmaceutical products (surfaces and coating layers of tablets, granular composition, phase analysis); i) morphological structures of textile materials; j) determination of sorts of damages to fibres (smelting, fibre rupture, tear/cut, fracture, etc.); k) expert examinations of biological materials - trichological material and its damage, shells of soil microorganisms for pedological forensic investigations, insect eggs to determine post mortem interval, etc.); l) intersecting lines of writing and print tools (superposition - writing tool x print toner); m) glass; n) fragments of building materials; o) fractures of materials (determination of the character of the fracture area); p) toolmark slipped impressions (forensic technical examinations, ballistics, unlike classic optical light microscopy facilitates more detailed comparisons);

Recently, dual systems with focused ion beam have considerably extended possibilities of electron microscopy. These systems are predominantly applied in the study of the inner structure of micro-and nanoparticles, layers and composites (intersecting lines in forensic graphical examinations, analyses of functional glass layers, colour variable pigments, etc.), the study of alloys microdefects, creating 3D models of particles and aggregates, and the possibility to study the inner structure of thermogenetic particles is also of key importance, etc.

Automated mineralogical analyses are a great asset of the system for the analysis of mineral phases, particularly soils. These are modified SEM systems that are equipped with 2-4 EDS detectors. Classification of minerals into individual classes is carried out on the basis of BSE signal and chemical composition. These systems are standardly used for polished this sections. The experiments that were conducted at the ICP proved that it is possible to work directly with untreated grains of mineral phases. Grain preparations from real soil samples, mineral fractions (approx. 200-600 $\mu \mathrm{m}$ ) were employed for testing. Grains were attached through a double- sided stub with carbon tape $26 \mathrm{~mm}$ in diameter, namely in one layer. The actual data collection was carried on mineral analyzer TIMA, on platform MIRA, using field-emission source of electrons. The device was equipped with two opposite fitted EDS 
detectors with an active area of $30 \mathrm{~mm} 2$. The data collection was undertaken in accelerating voltage of $25 \mathrm{kV}$ and current of $5 \mathrm{nA}$. The working distance amounted to $15 \mathrm{~mm}$ and the size of the microscope field of view was set at the value of $1450 \mu \mathrm{m}$. The threshold to differentiate mineral particles from the background in imaging by backscattered electrons was set to at level of $15 \%$. Measurements were carried out with the step of $10 \mu \mathrm{m}$. The spectrum of 1000 counts was collected from each point analyzed. Percentage rate of the unidentified phase was in all conducted experiments about 5-16\%, which is a satisfactory result for the majority of comparative tasks in a forensic field. The obtained quantitative data are subsequently processed through statistical methods.

Other identification options for differentiation of mineral phases hardly distinguishable by different analyses are provided by cathode luminescence, mainly colour one and an accurate quantitative measurement of their spectral characteristics using microspectrofotometer. The measured spectra usually show considerable variability in the intensity of luminescence (reflect differences in chemistry of phases, but also variable conditions of measurement). However, after standardization of the spectra, the differences within one sample are only minimal and it is possible to apply them for differentiation of otherwise identical phases.

Until recently, the analysis of the organic phase has brought big problems in multicomponent particles and composites by applying SEM. This question has been in principle unsolvable for e.g. analysis of postblast residues, when retrieval of the particles of $10 \mu \mathrm{m}$ in heavily contaminated sample by techniques that would allow the analysis of its organic component is not feasible.

One of the very interesting options appears to be the Raman spectrometry technique, which is nowadays available as a supplement to SEM EDX. Newly available is the device that is fully confocal, SEM keeps full functionality and scan range, very high resolution (for green laser resolution 360nm FWHD; 430nm Rayleigh), it is fitted with high quality objective lens, allows mapping through Raman spectrometry in a volume $250 \mu \mathrm{m}$ x $250 \mu \mathrm{m}$ x $250 \mu \mathrm{m}$ by piezo driven scanner (capacitive feedback linearized) and obtaining high quality white light image $(250 \mu \mathrm{m} \times 250 \mu \mathrm{m})$ immediately in the SEM chamber. This technique is currently undergoing intensive testing and it seems that the method could significantly help to solve problems with the analysis of organic phases in electron microscopy, not only in the case of post-blast residues and explosives.

\section{Summary}

The application of new techniques significantly extends possibilities of classic microscopic procedures in a forensic field and allows acquisition of needed quantitative data in the forensic analysis of pedological phases, discrimination of mineral phases or the option of analysis of the organic phase directly in the SEM chamber.

\section{References:}

[1] Kotrlý M., Turková I.: Proc. SPIE 9073, 2014, SPIE, 90730U-1 - 10, Vol. 9073, 2014.

[2] Jiruse J. et al.: Microsc. Microanal. 20 (Suppl 3), 990-991, 2014.

[3] Acknowledgements - microanalytical methods at ICP were supported by projects: VD20062008B10, VD20072010B15, VG20102015065, VF20112015016, VF20122015027. 